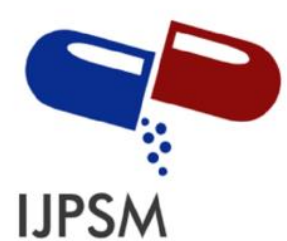

Patil Abhishek S. et al, Int. Journal of Pharmaceutical Sciences and Medicine (IJPSM),

Vol.6 Issue. 6, June- 2021, pg. 15-27

ISSN: 2519-9889

Impact Factor: 3.426

\title{
NIOSOMES: A PROMISING DRUG DELIVERY CARRIER
}

\section{Patil Abhishek S. ; Shaikh Bilal J.; Bhosale Ankush S.; Raut Indrayani D.; Nitalikar Manojkumar M. \\ Rajarambapu College of Pharmacy, Kasegaon (MS) India \\ Email id- patilabhishek056@gmail.com \\ DOI: 10.47760/ijpsm.2021.v06i06.002}

\author{
Correspondence Address: \\ Dr. Indrayani D. Raut \\ Assistant Professor, \\ Rajarambapu College of Pharmacy, Kasegaon (MS) India \\ Email id: idraut7363@gmail.com \\ Contact no. - 8600009718
}

\begin{abstract}
Niosomes are non-ionic surfactant-based multilamellar or unilamellar vesicles in which an aqueous solute solution is completely encapsulated by a membrane formed by surfactant macromolecules organized as a bilayer. Because vesicles are made up of a bilayer of non-ionic surface-active substances, the term "Niosomes" was coined (non-ionic surfactants). Niosomes are a unique drug delivery technology that encapsulates the medication in a vesicle. Ionic drug carriers are hazardous and inappropriate, whereas niosomal drug carriers are less dangerous. Niosomes do not require any specific handling or storage conditions. Niosomes have shown to be a promising drug carrier, with the potential to minimize medication side effects and improve therapeutic efficacy in a variety of disorders. Drug insolubility, instability, limited bioavailability, and fast degradation are all issues that niosomes address. The benefits, preparations, assessment, and medicinal uses of niosomes are discussed in this review article.
\end{abstract}

Keywords: Niosomes, non-ionic surfactants, ionic drug carriers, bioavailability, encapsulated, multilamellar 


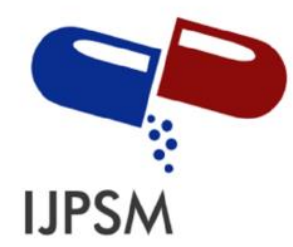

Patil Abhishek S. et al, Int. Journal of Pharmaceutical Sciences and Medicine (IJPSM), Vol.6 Issue. 6, June- 2021, pg. 15-27

ISSN: 2519-9889

Impact Factor: 3.426

\section{Introduction:}

Niosomes are multilamellar vesicles formed by hydration of synthetic nonionic surfactants with or without the addition of sterols such as cholesterol or other lipids. Niosomes are tiny lamellar structures made up of biodegradable, non-immunogenic, and biocompatible surfactants with a size range of 10 to $1000 \mathrm{~nm}$. The vesicles forming amphiphile in niosomes is a non-ionic surfactant that is normally stabilized by adding cholesterol and a little quantity of anionic surfactant such as dicetyl phosphate, which aids in vesicle stabilization. Niosomes have an architecture that combines hydrophilic and hydrophobic moieties, allowing them to accept medicinal molecules with a variety of solubilities. Niosomes can be used for a variety of drug delivery methods, including targeted, ocular, topical, parental, and so on. Niosomes have shown to be a promising drug carrier, with the potential to minimize medication side effects and improve therapeutic efficacy in a variety of disorders.

\section{Advantages:}

1. Niosomes are osmotically active and chemically stable..

2. They are non-immunogenic and biodegradable.

3. Their surface modification and formation are relatively simple.

4. Niosomes have a hydrophilic and hydrophobic architecture that allows them to accommodate medicinal molecules with a wide range of solubility.

5. Oral bioavailability of poorly absorbed medicines can be improved by niosomes.

6. They have structural qualities that are flexible and may be customized to fit the needs of the circumstance.

7. They have a high level of biological compatibility and are low in toxicity.

8. Niosomes can let medications penetrate deeper into the skin.

9. Niosomes change the organ distribution and metabolic stability of encapsulated drugs by prolonging their circulation.

10. They serve as a depot for short-acting peptide medicines, allowing the medicine to be released at a regulated rate

\section{Disadvantages:}

1. Due to fusion, aggregation, leakage of entrapped medicines, and hydrolysis of encapsulated medicines, the shelf life of niosome aqueous suspensions may be restricted.

2. Extrusion and sonication are two ways for preparing multilamellar vesicles that take time and may require specialized equipment for processing. 


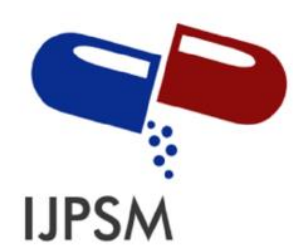

Patil Abhishek S. et al, Int. Journal of Pharmaceutical Sciences and Medicine (IJPSM), Vol.6 Issue. 6, June- 2021, pg. 15-27

ISSN: 2519-9889

Impact Factor: 3.426

3. The cost of such a medicine delivery system is significant.

4. Phospholipids can be oxidized and hydrolyzed at times.

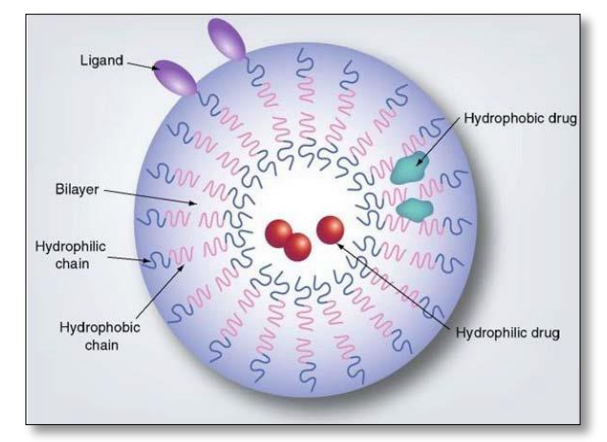

Figure 1: Structure of Niosome

\section{Types of Niosomes:}

Based on the vesicle size, niosomes can be divided into three groups [1]:

1. Small Unilamellar Vesicles (SUV) - Size $=0.025-0.05 \mu \mathrm{m}$ )

2. Multilamellar Vesicles (MLV) - Size $=>0.05 \mu \mathrm{m}$ )

3. Large Unilamellar Vesicles (LUV) - Size $=>0.10 \mu \mathrm{m}$ ).

\section{Compositions of niosomes:}

The two major components used for the preparation of niosomes are,

\section{Non-ionic surfactants:}

Selection of surfactant is done on the basis of HLB value. As Hydrophilic Lipophilic Balance (HLB) is a good indicator of the vesicle forming ability of any surfactant, HLB number in between 4 and 8 was found to be compatible with vesicle formation [2].

a) Alkyl ethers:

Some surfactants for the preparation of niosomes containing drugs/chemicals [3].

1) Surfactant-I (Mol.Wt.473) is C16 monoalkyl glycerol ether with average of three glycerol units.

2) Surfactant-II (Mol.Wt.972) is diglycerol ether with average of the seven glycerol units.

3) Surfactant III (Mol.Wt.393) is ester linked surfactant. 


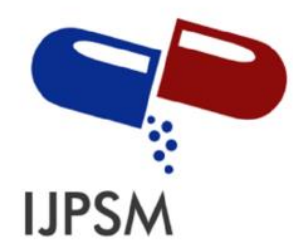

Patil Abhishek S. et al, Int. Journal of Pharmaceutical Sciences and Medicine (IJPSM), Vol.6 Issue. 6, June- 2021, pg. 15-27

ISSN: 2519-9889

Impact Factor: 3.426

\section{b) Alkyl esters:}

Sorbitan esters are most preferred surfactant used for the preparation of niosomes amongst this category of surfactants. Vesicles prepared by the polyoxy ethylene sorbitan monolaurate are relatively soluble than other surfactant vesicles]. For example, polyoxyethylene (polysorbate 60) has been utilized for encapsulation of diclofenac sodium [4].

c) Alkyl amides:

To make niosomal vesicles, alkyl amides (such as galactosides and glucosides) have been used.

d) Fatty acid and amino acid compounds:

Some niosomes have also been created with long chain fatty acids and amino acid moieties.

\section{Cholesterol:}

Steroids are key components of cell membranes, and their presence alters bilayer fluidity and permeability. Cholesterol is a steroid derivative utilized mostly in the manufacture of niosomes. Although it may not play a role in bilayer development, its relevance in niosome formation and modulation of layer properties cannot be overlooked. Incorporation of cholesterol alters niosome features such as membrane permeability, stiffness, encapsulation efficiency, the ease with which freeze-dried niosomes may be rehydrated, and their toxicity.

\section{Charged molecule:}

The addition of certain charged compounds to niosomes increases their stability by preventing coalescence through electrostatic repulsion. Diacetyl phosphate (DCP) and phosphotidic acid are the negatively charged compounds employed. These charged molecules are mostly utilized to inhibit niosome aggregation.

\section{Methods of preparation:}

\section{(A) Preparation of small unilamellar vesicles :}

\section{Sonication :}

A typical method of production of the vesicles is by sonication of solution as described by Cable. In this method an aliquot of drug solution in buffer is added to the surfactant/cholesterol mixture in a $10 \mathrm{ml}$ glass vial. The mixture is probe sonicated at $60^{\circ} \mathrm{C}$ for 3 minutes using a sonicator with a titanium probe to yield niosomes [5]. 


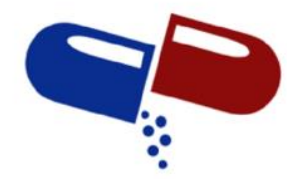

IJPSM

Patil Abhishek S. et al, Int. Journal of Pharmaceutical Sciences and Medicine (IJPSM), Vol.6 Issue. 6, June- 2021, pg. 15-27

ISSN: 2519-9889

Impact Factor: 3.426

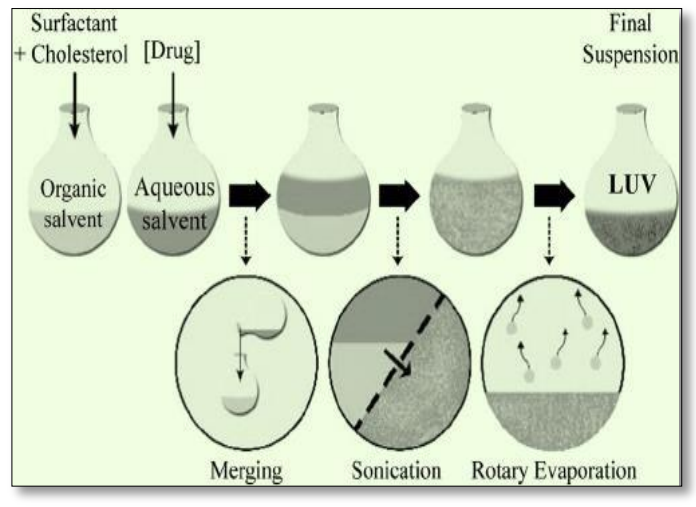

Figure 2: Sonication metho

\section{Microfluidization :}

In this method two fluidized streams move forward through precisely defined micro channel and interact at ultrahigh velocities within the interaction chamber. A common gateway is arranged such that the energy supplied to the system remains within the area of niosomes formation. The result is a greater uniformity, smaller size and better reproducibility [6].

\section{(B) Preparation of multilamellar vesicles :}

\section{Hand shaking method (Thin film hydration technique) :}

In this method, mixture of vesicles forming ingredients like surfactant and cholesterol are dissolved in a volatile organic solvent (diethyl ether, chloroform or methanol) in a round bottom flask. The organic solvent is removed at room temperature $\left(20^{\circ} \mathrm{C}\right)$ using rotary evaporator leaving a thin layer of solid mixture deposited on the wall of the flask. The dried surfactant film can be rehydrated with aqueous phase at $0-60^{\circ} \mathrm{C}$ with gentle agitation. This process leads to formation of typical multilamellar niosomes film of lipid on the wall of rotary flash evaporator. The aqueous phase containing drug was added slowly with intermittent shaking of flask at room temperature followed by sonication [7]. 


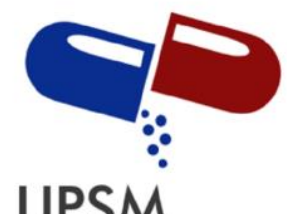

Patil Abhishek S. et al, Int. Journal of Pharmaceutical Sciences and Medicine (IJPSM), Vol.6 Issue. 6, June- 2021, pg. 15-27

ISSN: 2519-9889

Impact Factor: 3.426

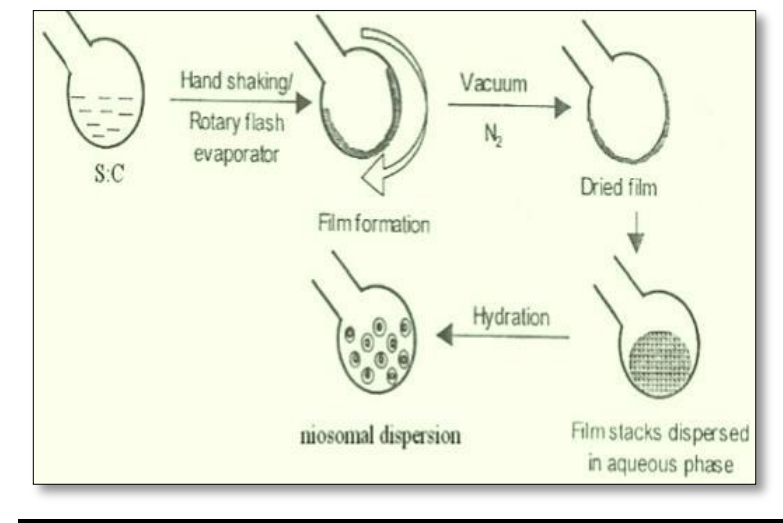

Figure 3: Hand shaking method

\section{Trans-membrane pH gradient (inside acidic) drug uptake process (remote loading):}

In this method surfactant and cholesterol are dissolved in chloroform. The solvent is then evaporated under reduced pressure to obtain a thin film on the wall of the round-bottom flask. The film is hydrated with $300 \mathrm{~mm}$ citric acid ( $\mathrm{pH}$ 4.0) by vortex mixing. The multilamellar vesicles are frozen and thawed three times and later sonicated. To this niosomal suspension, aqueous solution containing $10 \mathrm{mg} / \mathrm{ml}$ of drug is added and vortexed. This mixture is later heated at $60^{\circ} \mathrm{C}$ for 10 minutes to produce the desired multilamellar vesicles [8].

\section{(C) Preparation of large unilamellar vesicles :}

\section{Reverse Phase Evaporation Technique (REV):}

In this method Cholesterol and surfactant (1:1) are dissolved in a mixture of ether and chloroform. An aqueous phase containing drug is added to this and the resulting two phases are sonicated at $4-5^{\circ} \mathrm{C}$. The clear gel formed is further sonicated after the addition of a small amount of phosphate buffered saline (PBS). The organic phase is removed at $40^{\circ} \mathrm{C}$ under low pressure. The resulting viscous niosome suspension is diluted with PBS and heated on a water bath at $60^{\circ} \mathrm{C}$ for $10 \mathrm{~min}$ to yield niosomes [9].

\section{Ether injection method:}

This method is based on slow injection of niosomal ingredients in ether through a 14-gauge needle at the rate of approximately $0.25 \mathrm{ml} / \mathrm{min}$ into a preheated aqueous phase maintained at $60{ }^{\circ} \mathrm{C}$. Formation of larger unilamellar vesicles is due to slow vaporization of solvent results in an ether gradient extending towards the interface of aqueous-nonaqueous interface. The disadvantages of this method are that a small amount of ether is frequently present in the vesicles suspension and is difficult to remove [10]. 


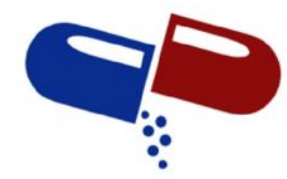

IJPSM

Patil Abhishek S. et al, Int. Journal of Pharmaceutical Sciences and Medicine (IJPSM), Vol.6 Issue. 6, June- 2021, pg. 15-27

ISSN: 2519-9889

Impact Factor: 3.426

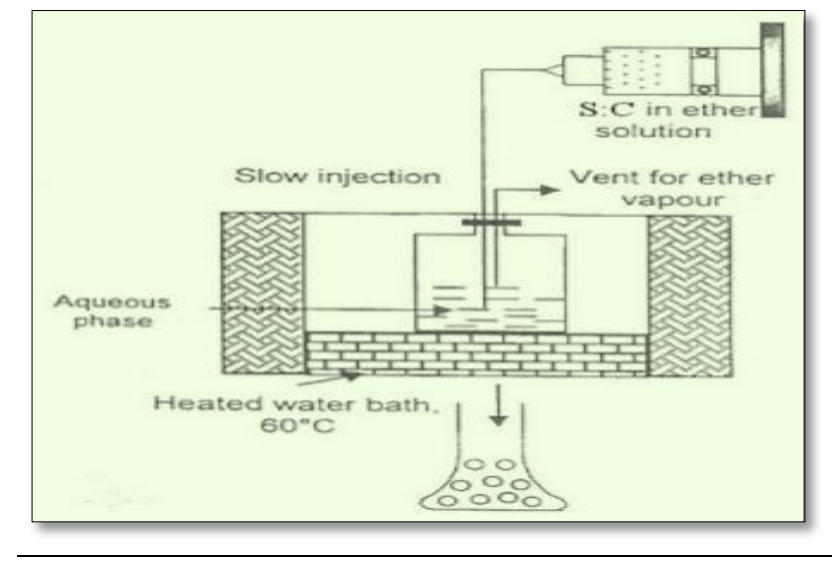

Figure 4: Ether injection method

\section{(D) Miscellaneous :}

\section{Multiple membrane extrusion method :}

Mixture of surfactant, cholesterol and dicetyl phosphate in chloroform is made into thin film by evaporation. The film is hydrated with aqueous drug solution and the resultant suspension extruded through polycarbonate membranes, which are placed in series for upto 8 passages. It is a good method for controlling niosome size [11].

\section{The bubble method:}

It is a one-step preparation of niosomes without the organic solvent. The bubbling unit consist of round bottom flak with three necks position in water bath to control the temperature Water cooled reflex and thermometer is positioned in the first and second neck and nitrogen supply through the third neck Cholesterol and surfactant are dispersed in this buffer $(\mathrm{pH} 7.4)$ at $70^{\circ} \mathrm{C}$ the dispersion mixed for 15 seconds with high shear homogenizer and immediately afterward bubble at $70^{\circ} \mathrm{C}$ using nitrogen gas [12]. 


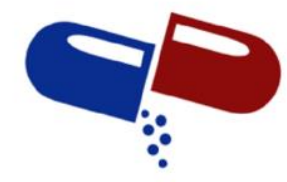

IJPSM

Patil Abhishek S. et al, Int. Journal of Pharmaceutical Sciences and Medicine (IJPSM), Vol.6 Issue. 6, June- 2021, pg. 15-27

ISSN: 2519-9889

Impact Factor: 3.426

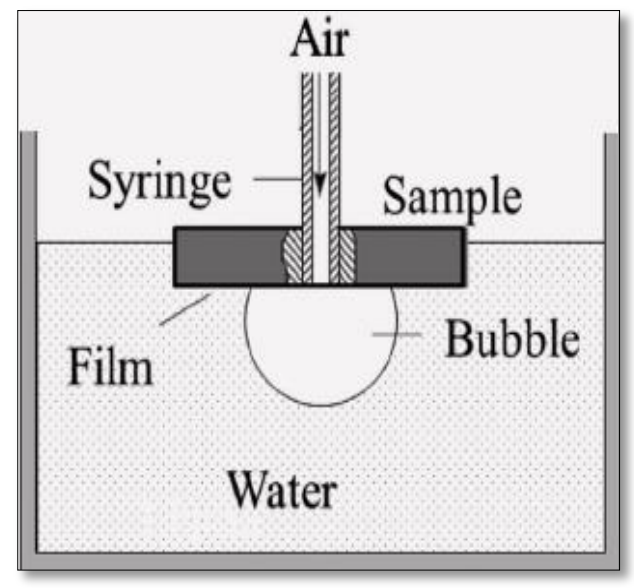

Figure 5: Bubble method

\section{Formation of niosomes from proniosomes :}

In this method water-soluble carrier such as sorbitol are coated with surfactant. The result of the coating process is a dry formulation. In which each water-soluble particle is covered with a thin film of dry surfactant [13]. This preparation is termed "Proniosomes". The niosomes are recognized by the addition of aqueous phase at T $>\mathrm{Tm}$ and brief agitation $(T=$ Temperature. $T m=$ mean phase transition temperature $)$

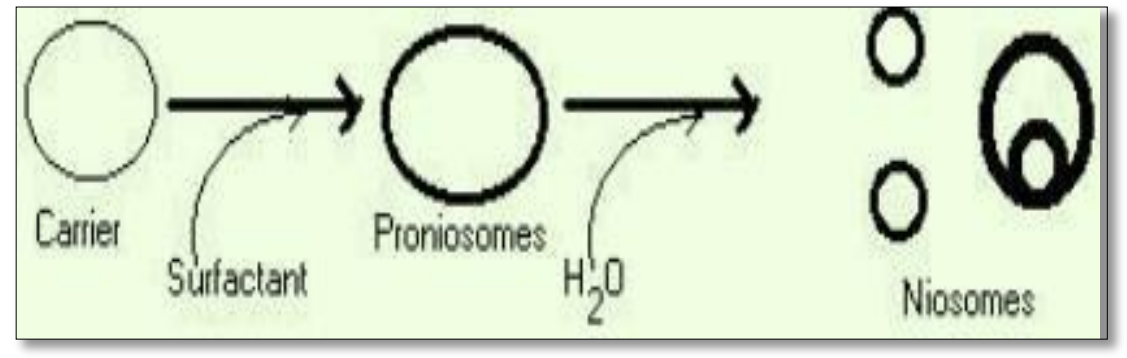

Figure 6: Formation of niosomes from proniosomes

\section{Separation of unentrapped drug:}

The removal of unentrapped solute from the vesicles can be accomplished by various techniques, which include:

1. Dialysis: The aqueous niosomal dispersion is dialyzed in dialysis tubing against phosphate buffer or normal saline or glucose solution [14].

2. Gel Filtration: The unentrapped drug is removed by gel filtration of niosomal dispersion through a Sephadex-G-50 column and elution with phosphate buffered saline or normal saline [15]. 


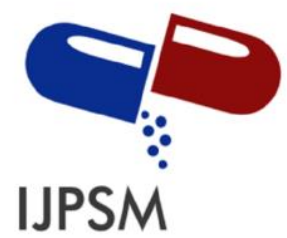

Patil Abhishek S. et al, Int. Journal of Pharmaceutical Sciences and Medicine (IJPSM), Vol.6 Issue. 6, June- 2021, pg. 15-27

ISSN: 2519-9889

Impact Factor: 3.426

\section{Factors affecting physico-chemical properties of niosomes:}

i. $\quad$ Nature of Surfactants :

A surfactant has a hydrophilic head and hydrophobic tail. The hydrophobic tail may consist of one or two alkyl or perfluoro alkyl groups or in some cases a single steroidal group. The ester type surfactants are chemically less stable than ether type surfactants and the former is less toxic than the latter due to esterlinked surfactant degraded by esterase's to triglycerides and fatty acid in vivo. The surfactants with alkyl chain length from C12-C18 are suitable for preparation of niosomes [16].

ii. Osmotic stress :

Addition of a hypertonic salt solution to a suspension of niosomes brings about reduction in diameter. In hypotonic salt solution, there is initial slow release with slight swelling of vesicles probably due to inhibition of eluting fluid from vesicles, followed by faster release, which may be due to mechanical loosening of vesicles structure under osmotic stress [17].

\section{iii. Temperature of Hydration :}

Hydration temperature influences the shape and size of the niosome. For ideal condition it should be above the gel to liquid phase transition temperature of system. Temperature change of niosomal system affects assembly of surfactants into vesicles and also induces vesicle shape transformation [18].

\section{iv. $\quad$ Nature of Encapsulated Drug :}

The drug interacts with surfactant head groups and develops the charge that creates mutual repulsion between surfactant bilayers and hence increases vesicle size. The aggregation of vesicles is prevented due to the charge development on bilayer [19].

\section{v. Membrane Composition :}

Niosomes can be prepared with addition of different additives along with surfactants and drugs. Addition of cholesterol molecule to niosomal system provides rigidity to the membrane and reduces the leakage of drug from niosome. Inclusion of cholesterol in niosomes increases its hydrodynamic diameter and entrapment efficiency. In general, the action of cholesterol is two folds; on one hand, cholesterol increases the chain order of liquid-state bilayers and on the other, cholesterol decreases the chain order of gel state bilayers. An increase in cholesterol content of the bilayers leads to decrease in the release rate of encapsulated material and therefore an increase of the rigidity of the bilayers obtained. Presence of charge tends to increase the interlamellar distance between successive bilayers in multilamellar vesicle structure and is responsible for increase in entrapped volume [20]. 


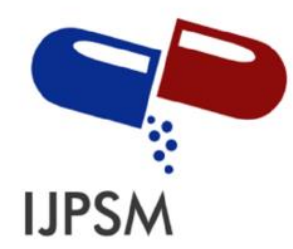

Patil Abhishek S. et al, Int. Journal of Pharmaceutical Sciences and Medicine (IJPSM), Vol.6 Issue. 6, June- 2021, pg. 15-27

ISSN: 2519-9889

Impact Factor: 3.426

\section{Method of preparation:}

Hand shaking method forms vesicles with greater diameter $(0.35-13 \mathrm{~nm})$ compared to the ether injection method (50-1000 nm). Small sized niosomes can be produced by Reverse Phase Evaporation method. Micro fluidization method gives greater uniformity and small size vesicles. Niosomes obtained by Trans membrane $\mathrm{pH}$ gradient (inside acidic) drug uptake process showed greater entrapment efficiency and better retention of drug [21].

\section{Evaluation parameters of niosomes:}

\section{Size :}

The mean diameter of niosomal vesicles may be estimated using the laser light scattering technique, and their shape is considered to be spherical. Electron microscopy, molecular sieve chromatography, ultracentrifugation, photon correlation microscopy, optical microscopy, and freeze fracture electron microscopy can also be used to assess the diameter of these vesicles.

\section{Bilayer formation :}

Under light polarisation microscopy, the assembly of non-ionic surfactants to create a bilayer vesicle is characterized by an X-cross formation.

\section{Membrane rigidity :}

The mobility of a fluorescence probe as a function of temperature can be used to determine membrane stiffness.

\section{Entrapment efficiency :}

Unentrapped drug is separated by dialysis, centrifugation, or gel filtration as described above, and the drug that remains entrapped in niosomes is determined by complete vesicle disruption with 50 percent n-propanol or 0.1 percent Triton X-100 and analyzing the resultant solution using the appropriate assay method for the drug.

\section{Number of lamellae :}

Nuclear magnetic resonance (NMR) spectroscopy, small angle X-ray scattering, and electron microscopy are used to determine this.

\section{In vitro release study :}

Several researchers have used dialysis tubing to conduct in vitro release rate studies. A dialysis sac is immersed in distilled water after being cleaned. The vesicle suspension is pipetted into and sealed in a bag made of tubing. The vesicles are then put in a $200 \mathrm{ml}$ buffer solution in a $250 \mathrm{ml}$ beaker and shaken constantly at $25^{\circ} \mathrm{C}$ or $37^{\circ} \mathrm{C}$. The drug content of the buffer is determined at various time intervals using an appropriate assay technique. 


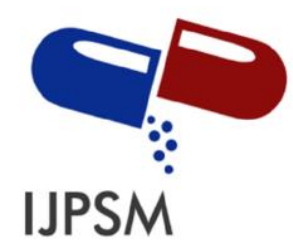

Patil Abhishek S. et al, Int. Journal of Pharmaceutical Sciences and Medicine (IJPSM), Vol.6 Issue. 6, June- 2021, pg. 15-27

ISSN: 2519-9889

Impact Factor: 3.426

\section{Applications:}

Niosomal technology has a wide range of applications and can be utilized to treat a variety of ailments.

\section{Niosomes as drug carriers :}

Iobitridol, a diagnostic chemical used in X-ray imaging, has also been carried by niosomes. Topical niosomes can operate as a solubilization matrix, a local depot for the prolonged release of dermally active substances, penetration enhancers, or a rate-limiting membrane barrier for drug absorption regulation.

\section{Drug targeting :}

The capacity of niosomes to target medications is one of its most beneficial features. Drugs can be targeted to the reticuloendothelial system using niosomes. Niosome vesicles are taken up preferentially by the reticuloendothelial system (RES). To target niosomes to certain organs, a carrier system (such as antibodies) can be linked to them (since immunoglobulins bind readily to the lipid surface of the niosome).

\section{Anti-neoplastic Treatment :}

The majority of anticancer medications have serious adverse effects. Niosomes have the ability to change metabolism, extend medication circulation and half-life, and thereby reduce pharmacological adverse effects. Niosomes cause a slower rate of tumour development and greater plasma levels, as well as longer clearance.

\section{Leishmaniasis :}

Leishmaniasis is a parasitic infection of the liver and spleen caused by a parasite of the genus Leishmania. The use of niosomes in experiments revealed that it was able to provide higher doses of the medicine without eliciting negative effects, allowing for increased therapeutic effectiveness.

\section{Niosomes as carriers for Hemoglobin :}

Hemoglobin can be transported via niosomes. The visible spectrum of niosomal suspension is superimposed over that of free haemoglobin. Vesicles are oxygen permeable, and the haemoglobin dissociation curve can be altered in the same way as non-encapsulated haemoglobin can.

\section{Transdermal delivery of drugs by niosomes :}

The main disadvantage of transdermal administration is the slow uptake of drugs via the skin. The transdermal distribution of medication integrated in niosomes resulted in a higher penetration rate.

\section{Anti-neoplastic Treatment :}

Anticancer medications have a lot of negative side effects. Niosomes have the ability to change metabolism, extend medication circulation and half-life, and thereby reduce pharmacological adverse effects. Niosomes reduce tumour growth and lead to greater plasma levels and delayed elimination. 


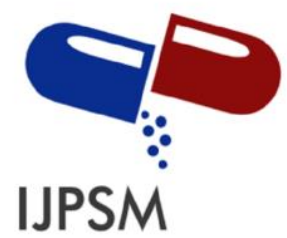

Patil Abhishek S. et al, Int. Journal of Pharmaceutical Sciences and Medicine (IJPSM), Vol.6 Issue. 6, June- 2021, pg. 15-27

ISSN: 2519-9889

Impact Factor: 3.426

\section{Ophthalmic drug delivery :}

Due to tear formation, corneal epithelial impermeability, non-productive absorption, and transitory residence duration, it is challenging to attain optimal bioavailability of drugs in ocular dosage forms such as ophthalmic solution, suspension, and ointment. However, medication bioavailability can be improved by using niosomal and liposomal delivery methods.

\section{Conclusion:}

Niosomes looked to be a popular drug delivery technology that was both stable and cost-effective. Niosomes have the potential to improve medication bioavailability and targeting, as well as reduce drug toxicity and adverse effects. Using innovative concepts like proniosomes, the niosome's drug delivery capability can be increased. Researchers agree that integrating the medicine into niosomes allows for improved targeting of the medicine to the proper tissue destination. Many different types of medications, such as toxic anti-cancer medications, anti-infective medications, anti-AIDS medications, anti-inflammatory medications, anti-viral medications, and so on, can be encapsulated.

\section{References}

[1]. Moghassemi S, Hadjizadeh A, Nano-niosomes as nanoscale drug delivery systems: an illustrated review, J. Cont. Rel. 2014, 185 (1), 22-36.

[2]. Liu T, Guo R, Hua W, Qiu J, Structure behaviors of hemoglobin in PEG 6000/Tween 80/Span 80/H2O niosome system. Colloids and Surfaces A: Physicochemical and Engineering Aspects. 2007, 293, 1-3, 255-261.

[3]. Bandyopadhyay P, Johnson M. Fatty alcohols or fatty acids as niosomal hybrid carrier: effect on vesicle size, encapsulation efficiency and in vitro dye release. Colloids and Surfaces B: Biointerfaces. 2007, 58, 68- 71.

[4]. Dingwoke Francis John, Yunus AA, Udokwu Japheth Chigbo, Ugwoke Sunday Paul, Ezeakulkenna. Tolnaftate loaded liposomes- design, and in-vitro evaluation. Universal Journal of Pharmaceutical Research. 2016; 1(2): 48-53.

[5]. Balasubramaniam A, Kumar VA, Pillai KS. Formulation and in-vivo evaluation of niosome encapsulated daunorubicin hydrochloride. Drug. Dev. Ind. Pharm. 2002; 28:1181-93.

[6]. Yoshioka T, Stermberg B, Florence AT. Preparation and properties of vesicles (niosomes) of sorbitan monoesters (Span 20, 40, 60, and 80) and a sorbitantriester (Span 85). Int. J. Pharm. 1994; 105:1-6.

[7]. Karki R, Mamatha GC, Subramanya G, Udupa N. Preparation, characterization and tissue disposition of niosomes containing isoniazid. Rasayan J Chem. 2008; 1:224-7.

[8]. Marianecci C, Rinaldi F, Marzio LD, Ciogli A, Esposito S, Carafa M. Polysorbate 20 vesicles as multi-drug carriers: in vitro preliminary evaluations,” Lett. Drug Design and Disc. 2013, 10(3), 212-218. 


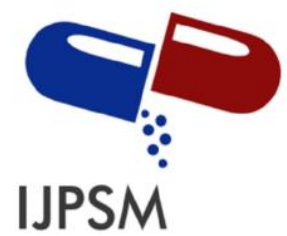

Patil Abhishek S. et al, Int. Journal of Pharmaceutical Sciences and Medicine (IJPSM), Vol.6 Issue. 6, June- 2021, pg. 15-27

ISSN: 2519-9889

Impact Factor: 3.426

[9]. Begum K, Khan AF, Hana HK, Sheak J, Jalil RU. Rifampicin niosome: preparations, characterizations and antibacterial activity against staphylococcus aureus and staphylococcus epidermidis isolated from acne," Dhaka University J. Pharm. Sci. 2015, 14, 1, 117-123.

[10].Manconi M, Sinico M, Valenti D, Lai F, Fadda AM, Niosomes as carriers for tretinoin: III. A study into the in vitro cutaneous delivery of vesicle-incorporated tretinoin,” Int. J. Pharm. 2006, 311, 1-2, 11-19.

[11]. Tavano L, Muzzalupo R, Mauro L, Pellegrino M, Andò S, Picci N, Transferrin-conjugated Pluronic niosomes as a new drug delivery system for anticancer therapy, Langmuir. 2013, 29, 41, 12638-12646.

[12]. Kumar GP, Rajeshwarrao P. Nonionic surfactant vesicular systems for effective drug delivery-an overview," ActaPharmaceuticaSinica B. 2011, 1(4), 208-219.

[13]. Zhang S, Morris ME. Efflux transporters in drug excretion. In: Wang B, Siahaan T, Soltero R (eds) Drug delivery: principles and applications. Wiley, Hoboken. 2005, 381-398.

[14].Gayatri Devi S, Venkatesh P and Udupa N. Niosomalsumatriptan succinate for nasal administration. Int. J. Pharm. Sci. 2000;62(6):479- 481.

[15]. R. Muzzalupo, L. Tavano, R. Cassano, S. Trombino, T. Ferrarelli, and N. Picci. A new approach for the evaluation of niosomes as effective transdermal drug delivery systems. Europ. J. Pharm. Biopharm. 2011, 79(1), 28-35.

[16].Ugochukwu AE, Nnedimkpa OJ, Rita NO. Preparation and characterization of Tolterodine tartrate proniosomes, Universal Journal of Pharmaceutical Research. 2017; 2(2): 22-25.

[17].Manosroi A, Wongtrakul P, Manosroi J. Characterization of vesicles prepared with various non-ionic surfactants mixed with cholesterol," Colloids and Surfaces B: Biointerfaces. 2003, 30, 1-2, 129-138.

[18].Ejiogu Deborah Chioma. Formulation and evaluation of etodolacniosomes by modified ether injection technique. Universal Journal of Pharmaceutical Research. 2016; 1(1): 1-6.

[19].Kapil Kumar, AK Rai. Proniosomes as a drug carrier for transdermal delivery of herbal drug, Journal of Pharmacy Research, 2012; 5(2):887-890.

[20]. Kumar GP, Rajeshwarrao P. Nonionic surfactant vesicular systems for effective drug delivery-an overview. Acta. Pharm. Sin. B. 2011, 1(4):208-219.

[21]. Yogesh Sharma, Kapil Kumar, SaiKrushnaPadhy. Formulation and evaluation of Atorvastatin calcium Niosomes, Int. J. Life. Sci. Scienti. Res., 2016, 2 (4), 1-4.

[22]. Lawrence J, Chauhan S, Lawrence SM, Barlow D. The formation, characterization and stability of non-ionic surfactant vesicles. STP Pharma Sci. 1996, 6(1):49-60. 